\title{
Erratum to: Phase Diagram of the ABC Model on an Interval
}

\author{
A. Ayyer · E.A. Carlen · J.L. Lebowitz · P.K. Mohanty • \\ D. Mukamel · E.R. Speer
}

Published online: 6 August 2011

(C) Springer Science+Business Media, LLC 2011

\section{Erratum to: J Stat Phys (2009) 137:1166-1204 DOI 10.1007/s10955-009-9834-x}

1. On page 1193, in the next to last line of the first paragraph of Sect. 9, the equation should $\operatorname{read} \beta<4 \pi / 3=(2 / 3 \sqrt{3}) \beta_{c}$.

2. In the following equations the quantity $\varkappa$ should be replaced by $1 / \varkappa$ : (A.8), the equation $f(y(t))=\operatorname{sn}\left(\varkappa t-F\left(\frac{1}{k}, k\right), k\right)$ directly above (A.9), (A.9), and (A.10).

3. In the first paragraph of the proof of Remark 5.1(c) (Appendix B, page 1202) the two inequalities on lines 1 and $3, \mathrm{~d} \tau_{K} / \mathrm{d} K>0$ and $\mathrm{d} \tau_{K(a)} / \mathrm{d} a>0$, respectively, should be

The online version of the original article can be found under doi:10.1007/s10955-009-9834-x.

A. Ayyer

Institut de Physique Théorique, IPhT, CEA Saclay, and URA 2306, CNRS, 91191

Gif-sur-Yvette Cedex, France

A. Ayyer

Present address:

Department of Mathematics, University of California, Davis, CA 95616, USA

E.A. Carlen · J.L. Lebowitz · E.R. Speer ( $\varangle)$

Department of Mathematics, Rutgers University, Piscataway, NJ 08854-8019, USA

e-mail: speer@math.rutgers.edu

J.L. Lebowitz

Department of Physics, Rutgers University, Piscataway, NJ 08854, USA

P.K. Mohanty

TCMP Division, Saha Institute of Nuclear Physics, 1/AF Bidhan Nagar, Kolkata 700064, India

D. Mukamel

Department of Physics of Complex Systems, Weizmann Institute of Science, Rehovot 76100, Israel 
reversed: $\mathrm{d} \tau_{K} / \mathrm{d} K<0$ and $\mathrm{d} \tau_{K(a)} / \mathrm{d} a<0$. Moreover in equations (B.6) and (B.8) the left hand sides should read $-\mathrm{d} \tau_{K} / \mathrm{d} a$ and the overall factor of 4 on the right hand sides should be replaced by 2 . 